COMMUNICATIONS ARISING HIV in Africa

\section{Chemokine-receptor genes and AIDS risk}

chliekelman et al. ${ }^{1}$ have provided a model to quantify the speed at which HIV-resistance haplotypes can become enriched in a susceptible population through a delay in the onset of AIDS, permitting greater lifetime reproduction and the selection of AIDS-delaying haplotypes. But we question their conclusion ${ }^{1}$ that there could be a rapid evolution of resistance to AIDS onset in some African populations if the current HIV epidemic persists, as this depends on an untested assumption: that variant forms of the chemokine-receptor-5 (CCR5) gene impart selective advantages or disadvantages in Africa that are comparable to those reported for African Americans ${ }^{2-6}$. Here we test this premise in a large Ugandan population, and find that CCR5 variants are not associated with HIV/AIDS disease risk in Africa - the origin and centre of the current AIDS pandemic. This gene may therefore not be subject to rapid evolutionary change as a result of the HIV epidemic in Africa.

We genotyped 1,149 Ugandan HIV-1positive individuals with previously measured CD4 counts $^{7}$ at the CCR5 locus. No CCR5 promoter allele was associated either with infection in a case-control study or with disease progression to death in a Cox regression analysis (Tables 1,2 ). Neither the guanine-thymine substitution at position -2554 , which characterizes the HHD and HHC haplotypes associated with maximal disease progression in African Americans ${ }^{2}$, nor the guanine-adenine change at position -2459 , which is consistently associated with accelerated progression in Caucasians ${ }^{3,6,8,9}$, was significantly associated with the rate of disease progression to death in Uganda.

We derived haplogroups and haplotypes from the different CCR5-promoter genotypes $^{2}$; analysis of 440 individuals with ascertainable haplogroups showed no overall correlation with the rate of disease
Table 1 Allele frequencies of CCR5 and CCR2 variants in HIV-positive and uninfected individuals

\begin{tabular}{|c|c|c|c|c|c|c|c|c|}
\hline & CCR2 64I & $-2733 G$ & $-2554 T$ & $-2459 G$ & $-2135 C$ & $-2132 T$ & $-2086 G$ & $-1835 T$ \\
\hline $\begin{array}{l}\text { Allele frequency } \\
\text { (HIV-positive) }\end{array}$ & 0.203 & 0.145 & 0.163 & 0.468 & 0.424 & 0.12 & 0.047 & 0.346 \\
\hline $\begin{array}{l}\text { Allele frequency } \\
\text { (controls) }\end{array}$ & 0.214 & 0.124 & 0.183 & 0.464 & 0.456 & 0.121 & 0.052 & 0.317 \\
\hline $\begin{array}{l}P \text { value } \\
\text { (allelic comparison, d.f. }=1 \text { ) }\end{array}$ & 0.59 & 0.3 & 0.26 & 0.87 & 0.26 & 0.94 & 0.67 & 0.21 \\
\hline $\begin{array}{l}P \text { value } \\
\text { (genotypic comparison, d.f. }=2 \text { ) }\end{array}$ & 0.25 & 0.5 & 0.07 & 0.85 & 0.26 & 0.73 & 0.88 & 0.19 \\
\hline $\begin{array}{l}P \text { value } \\
\text { (Cox regression, d.f. }=2 \text { ) }\end{array}$ & 0.34 & 0.28 & 0.28 & 0.365 & 0.94 & 0.70 & 0.60 & 0.18 \\
\hline
\end{tabular}

Allele frequencies and $P$ values for promoter single-nucleotide polymorphisms ${ }^{2}$ in CCR5 in HIV-positive cases and uninfected controls. $P$ values denote the significance of results. Cox modelling of a possible effect on the rate of disease progression to death included the genotype, ethnic group (Bantu or non-Bantu) and CD4 count at entry into the study. progression. In particular, the haplogroup $\mathrm{HHA} / \mathrm{HHF}^{\star} 2$, which is most clearly associated with disease retardation in African Americans ${ }^{2}$, was the most prevalent haplogroup in Ugandans but was not associated with altered disease progression.

Furthermore, the valine-isoleucine amino-acid change at position 64 of CCR2, which is in linkage disequilibrium with the CCR5 promoter and has been associated with delayed disease progression ${ }^{4,10}$, was not associated with disease retardation in Ugandans (see www.well.ox.ac.uk/hill/ramaley). We also examined nine CCR5 codingregion variants ${ }^{11-13}$, and found variation at only five sites, three of which have a variant allele frequency of $<0.1 \%$. None of these sites was associated with either HIV infection or rate of disease progression (see www.well.ox.ac.uk/hill/ramaley).

Our examination of over 1,100 HIV-positive and over $300 \mathrm{HIV}$-negative Ugandans represents the largest study of the genetics of HIV-1 susceptibility in an African population to date. The alleles and haplotypes identified in North America and Europe as conferring resistance or susceptibility to HIV are not associated with altered susceptibility to infection or altered rate of disease progression in this African population.

There are several factors that differ between these regions and which may account for these findings. In Uganda, the predominant HIV-1 clades are A and D as opposed to B in Europe and North America - and transmission is predominantly heterosexual, unlike in almost all published

\begin{tabular}{|c|c|c|c|c|c|c|}
\hline & \multirow[t]{2}{*}{ CD4 count } & \multirow[t]{2}{*}{$\mathrm{P}$ value } & \multirow[t]{2}{*}{$\beta$} & \multirow[t]{2}{*}{$\operatorname{Exp}(\beta)$} & \multicolumn{2}{|c|}{$95 \%$ confidence interval for $\exp (\beta)$} \\
\hline & & & & & Lower & Upper \\
\hline $\mathrm{HHA} / \mathrm{HHE}$ & 49 & 0.172 & -0.556 & 0.574 & 0.259 & 1.273 \\
\hline $\mathrm{HHA} / \mathrm{HHF} 2$ & 49 & 0.619 & -0.186 & 0.83 & 0.398 & 1.729 \\
\hline HHE/HHF2 & 28 & 0.621 & -0.205 & 0.815 & 0.361 & 1.837 \\
\hline HHF2/HHG1 & 26 & 0.111 & -0.685 & 0.504 & 0.217 & 1.17 \\
\hline $\mathrm{HHF} 2 / \mathrm{HHF} 2$ & 25 & 0.109 & -0.722 & 0.486 & 0.201 & 1.174 \\
\hline $\mathrm{HHA} / \mathrm{HHD}$ & 24 & 0.341 & -0.403 & 0.668 & 0.291 & 1.533 \\
\hline $\mathrm{HHA} / \mathrm{HHA}$ & 23 & 0.402 & -0.361 & 0.697 & 0.3 & 1.621 \\
\hline $\mathrm{HHE} / \mathrm{HHF} 1$ & 23 & 0.648 & 0.194 & 1.214 & 0.529 & 2.785 \\
\hline HHA/HHF1 & 20 & 0.728 & 0.151 & 1.163 & 0.498 & 2.717 \\
\hline $\mathrm{HHA} / \mathrm{HHG} 1$ & 20 & 0.443 & -0.402 & 0.669 & 0.24 & 1.866 \\
\hline
\end{tabular}

Cox-regression results include ethnic group (Bantu or non-Bantu), CD4 count at entry into the study, and the 10 most common CCR5-promoter haplogroup genotypes ${ }^{2}$. $P>0.05$ in all cases and the $95 \%$ confidence intervals for $\exp (\beta)$ span 1.0 . studies of the genetics of HIV/AIDS susceptibility. Furthermore, the types of opportunistic and chronic infection found in HIV-positive individuals in Africa differ significantly from those in Europe and North America. Africans also show more genetic diversity than Caucasians, and these two groups show differences in genotype frequencies at loci that may interact epistatically with CCR 5 variants.

Whatever the mechanisms that underlie this interpopulation heterogeneity, our findings call for caution in extrapolating associations between immunogenetic factors and infectious disease from one population to another. They also indicate that inferences regarding the influence of natural selection by HIV-1 on CCR5 genetic diversity in Africa ${ }^{1}$ need revision.

Patricia A. Ramaley , Neil French $\dagger$, Pontiano Kaleebu $\neq$, Charles Gilks $\dagger$, ${ }^{\star}$ Wellcome Trust Centre for Human Genetics, University of Oxford, Roosevelt Drive,

Oxford OX3 7BN, UK

e-mail:adrian.hill@well.ox.ac.uk

$\dagger$ Liverpool School of Tropical Medicine,

Pembroke Place, Liverpool L3 5QA, UK

$\ddagger$ Uganda Virus Research Institute/Medical Research Council (UK) Programme on HIV/AIDS,

PO Box 49, Entebbe, Uganda

\footnotetext{
1. Schliekelman, P., Garner, C. \& Slatkin, M. Nature 411, 545-546 (2001).

2. Gonzalez, E. et al. Proc. Natl Acad. Sci. USA 96, 12004-12009 (1999).

3. Martin, M. P. et al. Science 282, 1907-1911 (1998).

4. Mummidi, S. et al. Nature Med. 4, 786-793 (1998).

5. Kostrikis, L. G. et al. J. Virol. 73, 10264-10271 (1999).

6. An, P. et al. AIDS 14, 2117-2122 (2000).

7. French, N. et al. Lancet 355, 2106-2111 (2000).

8. McDermott, D. H. et al. Lancet 352, 866-870 (1998).

9. Clegg, A. O. et al. AIDS 14, 103-108 (2000).

10. Smith, M. et al. Science 277, 959-965 (1997).

11. Ansari-Lari, M. A., Liu, X. M., Metzker, M. L., Rut, A. R. \& Gibbs, R. A. Nature Genet. 16, 221-222 (1997).

12. Carrington, M. et al. Am. J. Hum. Genet. 61, 1261-1267 (1997). 13. Petersen, D. C. et al. AIDS 15, 171-177 (2001).
}

brief communications is intended to provide a forum both for brief, topical reports of general scientific interest and for technical discussion of recently published material of particular interest to non-specialist readers. Priority will be given to contributions that have fewer than 500 words, 10 references and only one figure. Detailed guidelines are available on Nature's website (www.nature.com) or on request from nature@nature.com James Whitworth $\ddagger$, Adrian V. S. Hill ${ }^{\star}$ 